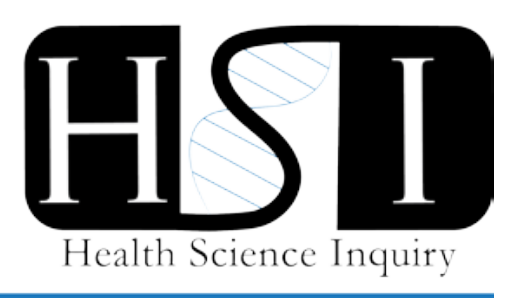

\title{
LINE-1 DNA methylation as a biomarker of early carcinogenesis
}

\author{
Priyanka Gogna \\ Department of Public Health Sciences, Queen's University
}

After sequencing of the human genome in 2003, science turned to the epigenome to improve understanding of the biological processes that dictate how the genetic code is read [1]. The interplay between the genome and epigenome can be described as the union of nature and nurture - while the genetic code remains fixed by nature, the epigenome may be altered. The epigenome is a set of heritable, yet modifiable chemical changes that affect the transcription, translation, and silencing of the genome [1]. The pattern of the epigenome is responsible for gene activation, silencing, and facilitation of tissue-specific gene expression in cells of the human body. Mutations leading to genetic changes in DNA are considered rare, and typically occur in the context of disease processes such as cancer [1]. However, the epigenome is susceptible to change due to environmental factors, and is expected to change across an individual's lifetime [2]. This key characteristic of the epigenome has made it an important topic in chronic disease research [3]. DNA methylation, histone modification, nucleosome modeling, and gene expression regulation by microRNA (miRNA) are known epigenetic regulation mechanisms [3]. This paper explores the potential for DNA methylation to serve as a biomarker of early carcinogenesis.

DNA methylation is a widely studied epigenetic modification in the field of molecular epidemiology, which seeks to understand molecular processes by which known risk factors may cause disease in the human body. Molecular epidemiology techniques are commonly applied to research focused on the etiology of cancer, where long latency periods make it difficult to study the effect of toxins on cancer risk directly. Breaking down the disease continuum allows molecular epidemiologists to study the mechanistic relationships by which lifestyle exposures can lead to disease. For example, to elucidate the mechanism by which body mass index (BMI) may lead to increased colorectal cancer risk, an investigator may study the relationship between BMI and DNA methylation - a known precursor to cancer.

Another consideration of molecular epidemiology research involves the investigation of genetic factors that affect the molecular processes under study. The efficiency of molecular processes in the cell is influenced by an individual's genetics [4]. By incorporating genetic factors into the study of molecular epidemiology, it becomes possible to target those individuals that are at an increased risk of disease due to genetic polymorphisms. When data on lifestyle exposures and genetic factors are combined, a more complete picture of the risk of chronic diseases begins to form. This research can then be directly used to test individuals for genetic variants, as well as create targeted interventions for those at an increased risk. Specifically, genetic screening and development of drugs that may help mitigate deficiencies associated with methylation processes could benefit individuals determined to be at increased risk [5]. In any case, molecular epidemiology allows intervention research to move forward with greater information regarding the biological mechanisms behind disease causation [5].

Biomarkers have become important surrogate endpoints of studies in the quest for improved disease understanding due to their ease of measurement and earlier detection of disease [6]. In a recent review, Strimbu and Tavel define biomarkers as objective indications of medical state observed from outside the patient - which can be measured accurately and reproducibly [6]. Long interspersed nuclear element-1 (LINE-1) DNA methylation is a specific form of DNA methylation, and an established precursor to carcinogenesis. It is specifically linked to colorectal, breast, cervical, head and neck, and kidney cancer, as well as all-cancer mortality, and other chronic diseases [7, 8]. Although the exact role of LINE-1 DNA methylation is not clear, hypomethylation of LINE-1 elements is linked to genomic instability, a known precursor to cancer [3]. Furthermore, LINE-1 DNA methylation is a relatively stable measure, and does not change over shorter time periods [9]. This is an essential component of a biomarker, as a measure with poor stability and daily or monthly fluctuations would be difficult to quantify without consistent measurement and follow-up.

In recent years, microchip-based approaches have been used to quantify DNA methylation over traditional polymerase chain reaction (PCR) based approaches [10]. This approach is able to quantify methylation with 
improved reliability and validity over previous methods [10]. It is perhaps these types of advancements in cheaper, easier to use measurement tools that will further propel the use of LINE-1 DNA methylation as a common biomarker for detecting carcinogenesis in its early stages. Basic science studies show that there is biological probability for LINE-1 DNA methylation to lie on the causal pathway between specific lifestyle factors and carcinogenesis [7, 8]. Although some current research suggests that LINE-1 DNA methylation is tissue-specific, methylation measured in leukocyte cells is also associated with colorectal, bladder, and breast cancer risk [8].

Combined, these aspects of LINE-1 DNA methylation demonstrate its potential as a biomarker of early carcinogenesis. Currently, the relationship between the majority of cancer risk factors and LINE-1 DNA methylation has not been established. However, epidemiologic studies investigating these relationships continue to provide new research, allowing for potential future implementation of LINE-1 DNA methylation as a biomarker of early carcinogenesis in clinical work.

\section{References}

[1] Moore, L. D., Le, T., Fan, G. (2012) DNA Methylation and Its Basic Function. Neuropsychopharmacology, 38 (1), 2338.

[2] Bollati, V., Schwartz, J., Wright, R., Litonjua, A., Tarantini, L., Suh, H., et al. (2009) Decline in genomic DNA methylation through aging in a cohort of elderly subjects. Mechanisms of Ageing and Development, 130 (4), 234239.
[3] Verma, M., Rogers, S., Divi, R. L., Schully, S. D., Nelson, S., Joseph Su, L., et al., (2014) Epigenetic Research in Cancer Epidemiology: Trends, Opportunities, and Challenges. Cancer Epidemiology Biomarkers \& Prevention, 23 (2), 223233.

[4] Meaney, M. J. (2010) Epigenetics and the biological definition of gene $x$ environment interactions. Child Dev., 81 (1), 4179.

[5] Ehrlich, M. (2009) DNA hypomethylation in cancer cells. Epigenomics, 1 (2), 239259.

[6] Strimbu, K., Tavel, J. A. (2010) What are biomarkers? Current Opinion in HIV and AIDS, 5 (6), 463436.

[7] Joyce, B. T., Gao, T., Zheng, Y., Liu, L., Zhang, W., Dai, Q., et al. (2016) Prospective changes in global DNA methylation and cancer incidence and mortality. Nature, 115 (4), 465472.

[8] 8 Terry, M. B., Delgado-Cruzata, L., Vin-Raviv, N., Wu, H. C., Santella, R.M. (2011) DNA methylation in white blood cells. Epigenetics, 6 (7), 828837.

[9] Kok, R. M., Smith, D. E. C., Barto, R., Spijkerman, A. M. W., Teerlink, T., Gellekink, H. J., et al., (2007) Global DNA methylation measured by liquid chromatography-tandem mass spectrometry: analytical technique, reference values and determinants in healthy subjects. Clin Chem Lab Med., 45 (7), 903911.

[10] Pidsley, R., Zotenko, E., Peters, T. J., Lawrence, M. G., Risbridger, G. P., Molloy, P., et al. (2016) Critical evaluation of the Illumina Methylation EPIC BeadChip microarray for whole-genome DNA methylation profiling. Genome Biology, 6, 117.

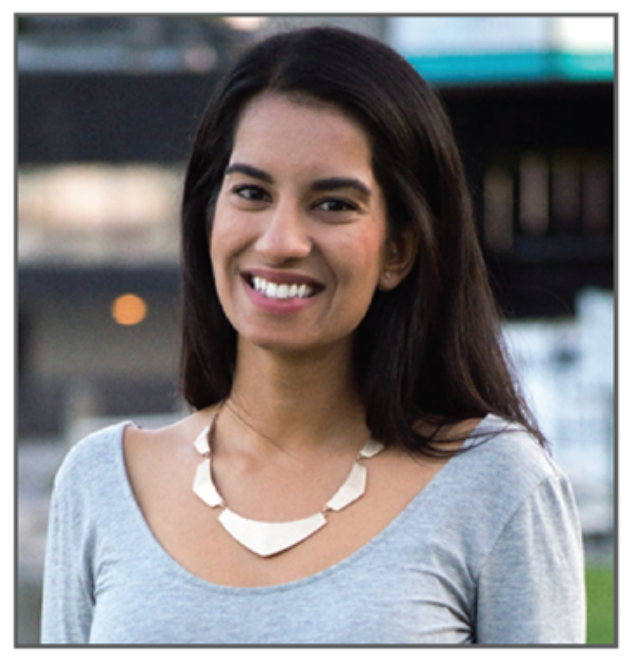

Priyanka Gogna is a 2nd year MSc in epidemiology candidate in the Department of Public Health Sciences at Queen's University. Her thesis research is on the relationship between colorectal cancer risk factors and DNA methylation in healthy colon tissue. Her research interests include environmental and cancer epidemiology. 